\title{
Calibration and Distortion Field Compensation of Gradiometer and the Improvement in Object Remote Sensing
}

\author{
Xiangchao $\mathrm{Hu}^{1,3}$, Hongfeng Pang ${ }^{1,2,3}$, Liangrui $\mathrm{Fu}^{1}$, Dixiang $\mathrm{Chen}^{3}$ and Mengchun $\mathrm{Pan}^{3}$ \\ ${ }^{1}$ Northwest Institute of Nuclear Technology, Xi'an, China \\ ${ }^{2}$ Academy of Equipment, Beijing, China \\ ${ }^{3}$ National University of Defense Technology, Changsha, China
}

\begin{abstract}
Magnetometer, misalignment error and distortion field can reduce the accuracy of gradiometers. So, it is important to calibrate and compensate gradiometers error. Scale factor, bias, nonorthogonality, misalignment and distortion field should be considered. A gradiometer is connected by an aluminium frame, which contains two fluxgate magnetometers. A nonmagnetic rotation equipment is used to change gradiometer attitude, and the compensation parameters are estimated. Experiment results show that, after calibration and compensation, error of each axis is reduced from $888.4 \mathrm{nT}, 1292.6 \mathrm{nT}$ and $168.9 \mathrm{nT}$ to $15.3 \mathrm{nT}, 22.1 \mathrm{nT}$ and $9.9 \mathrm{nT}$, respectively. It shows that the proposed method can calibrate gradiometer and compensate distortion field. After calibration and compensation, the object remote sensing performance is improved.
\end{abstract}

\section{Introduction}

Gradiometers are widely used for unexploded ordinance (UXO) detection, underwater exploration, and geologic research [1,2]. Magnetometer error, misalignment and distortion field influence the accuracy of gradiometers. It is important to calibrate gradiometer error and compensate distortion field. Scale factor, bias, nonorthogonality, and misalignment are hard to solve by mechanism [3,4]. In geomagnetic field circumstance, $0.1^{\circ}$ misalignment error will lead to about $100 \mathrm{nT}$ vector measurement error. Also, distortion field is hard to be avoided when it is deployed in vehicles [5,6]. Recently, magnetometer calibration has been developed using estimation methods [7-11]. However, there is little work in gradiometer calibration and compensation.

In this paper, a fluxgate gradiometer is designed, and an integrated calibration and compensation method is proposed. The true value of geomagnetic field intensity is obtained by a proton magnetometer. The nonmagnetic rotation equipment is used to change gradiometer attitude and the compensation parameters are estimated accurately. After integrated compensation, the measurement error of gradiometer is reduced obviously. In addition, the compensated gradiometer can be more effective for magnetic abnormity measurement.

\section{Calibration and compensation theory}

\subsection{Magnetometer calibration and distortion field compensation}

Considering of different scale factors, bias errors and nonorthogonality, the output of a three axis magnetometer can be expressed as [8]:

$$
B_{k}=D\left(H_{k}+b^{\prime}\right)+\varepsilon_{k}
$$

Where $H_{k}$ is the measurement of an ideal three axis magnetometer. $B_{k}$ is the measurement of nonorthogonal three axis magnetometer. $b^{\prime}=\left[\begin{array}{lll}b_{x}^{\prime} & b_{y}^{\prime} & b_{z}^{\prime}\end{array}\right]$ is bias vector. $D=\left[K_{x} D_{x y} D_{x z} ; D_{x y} K_{y} D_{y z} ; D_{x z} D_{y z} K_{z}\right]$ represent scale factor and nonorthogonality. $\varepsilon_{k}$ are measurement noise; $k$ is measurement point. Thus, the calibration model can be expressed as [12]: Considering of distortion field, the error model can be expressed as:

$$
B_{k}=P D\left(H_{k}+b^{\prime}\right)+b_{H}+\varepsilon_{k}
$$

Where $P$ is soft material distortion field matrix, and $b_{H}$ is hard material distortion field matrix. (2) can be expressed as:

$$
\begin{aligned}
{\left[\begin{array}{l}
B_{k}^{x} \\
B_{k}^{y} \\
B_{k}^{z}
\end{array}\right]=} & {\left[\begin{array}{lll}
p_{11} & p_{12} & p_{13} \\
p_{21} & p_{22} & p_{23} \\
p_{31} & p_{32} & p_{33}
\end{array}\right] \cdot\left[\begin{array}{lll}
D_{11} & D_{12} & D_{13} \\
D_{21} & D_{22} & D_{23} \\
D_{31} & D_{32} & D_{33}
\end{array}\right] } \\
& \cdot\left[\begin{array}{l}
H_{k}^{x}+b_{x}^{\prime} \\
H_{k}^{y}+b_{y}^{\prime} \\
H_{k}^{z}+b_{z}^{\prime}
\end{array}\right]+\left[\begin{array}{l}
b_{H}^{x} \\
b_{H}^{y} \\
b_{H}^{z}
\end{array}\right]+\left[\begin{array}{c}
\varepsilon_{k}^{x} \\
\varepsilon_{k}^{y} \\
\varepsilon_{k}^{z}
\end{array}\right]
\end{aligned}
$$

(3) can be expressed as:

$$
\left[\begin{array}{l}
B_{k}^{x} \\
B_{k}^{y} \\
B_{k}^{z}
\end{array}\right]=\left[\begin{array}{lll}
q_{11} & q_{12} & q_{13} \\
q_{21} & q_{22} & q_{23} \\
q_{31} & q_{32} & q_{33}
\end{array}\right]\left[\begin{array}{l}
H_{k}^{x}+b_{x}^{\prime} \\
H_{k}^{y}+b_{y}^{\prime} \\
H_{k}^{z}+b_{z}^{\prime}
\end{array}\right]+\left[\begin{array}{c}
b_{H}^{x} \\
b_{H}^{y} \\
b_{H}^{z}
\end{array}\right]+\left[\begin{array}{c}
\varepsilon_{k}^{x} \\
\varepsilon_{k}^{y} \\
\varepsilon_{k}^{z}
\end{array}\right]
$$


(4) can be expressed as:

$$
B_{k}=q H_{k}+b+\varepsilon_{k}
$$

(5) can be transformed as:

$$
H_{k}=Q\left(B_{k}-b\right)
$$

A further relationship can be obtained by squaring both sides of (6):

$$
H_{k}^{2}=\left[Q\left(B_{k}-b\right)\right]^{T}\left[Q\left(B_{k}-b\right)\right]
$$

$Q$ can be decomposed into an unitary matrix $Q_{A}$ and upper triangular matrix $Q_{R}$. (7) can be expressed as:

$$
H_{k}^{2}=\left[Q_{R}\left(B_{k}-b\right)\right]^{T}\left[Q_{R}\left(B_{k}-b\right)\right]
$$

Where $H_{k}$ is the true value of geomagnetic field intensity. Equation (8) can be transformed as:

$$
\begin{aligned}
H_{k}^{2}= & \left(B_{k}^{x}\right)^{2} M_{1}+\left(B_{k}^{y}\right)^{2} M_{2}+\left(B_{k}^{z}\right)^{2} M_{3} \\
& +\left(B_{k}^{x}\right)\left(B_{k}^{y}\right) M_{4}+\left(B_{k}^{y}\right)\left(B_{k}^{z}\right) M_{5}+\left(B_{k}^{z}\right)\left(B_{k}^{x}\right) M_{6} \\
& +\left(B_{k}^{x}\right) M_{7}+\left(B_{k}^{y}\right) M_{8}+\left(B_{k}^{z}\right) M_{9}+M_{10}
\end{aligned}
$$

Where (9) is a nonlinear equation. $M_{1}, \cdots M_{10}$ are expressed in [12]. There are nine unknown parameters to be estimate. There are $\mathrm{N}$ nonlinear equations when measurement point is $\mathrm{N}$. Nine unknown parameters can be calculated by solving nonlinear equations. Also, the parameters can be estimated by nonlinear least square [13].

\subsection{Misalignment calibration between magnetometers}

After magnetometer calibration and compensation, the two magnetometers can be considered as orthogonal. Misalignment error is the coordinate error between magnetometers. Magnetic field projection on the first and second magnetometer coordinate are $B_{1}=\left[B_{x 1}, B_{y 1}, B_{z 1}\right]^{T}$ and $B_{2}=\left[B_{x 2}, B_{y 2}, B_{z 2}\right]^{T}$ respectively. As shown in Figure1, the relationship of projection can be expressed as [14]:

$$
B_{1}=T_{1} T_{2} T_{3} B_{2}
$$

Where

$$
\begin{aligned}
& T_{1}=\left[\begin{array}{ccc}
\cos \psi & 0 & -\sin \psi \\
0 & 1 & 0 \\
\sin \psi & 1 & \cos \psi
\end{array}\right] \\
& T_{2}=\left[\begin{array}{ccc}
1 & 0 & 0 \\
0 & \cos \phi & \sin \phi \\
0 & -\sin \phi & \cos \phi
\end{array}\right] \\
& T_{3}=\left[\begin{array}{ccc}
\cos \theta & \sin \theta & 0 \\
-\sin \theta & \cos \theta & 0 \\
0 & 0 & 1
\end{array}\right]
\end{aligned}
$$

Where $\theta, \psi, \phi$ are misalignment angles. The misalignment angles can be calculated by solving nonlinear equations [12].

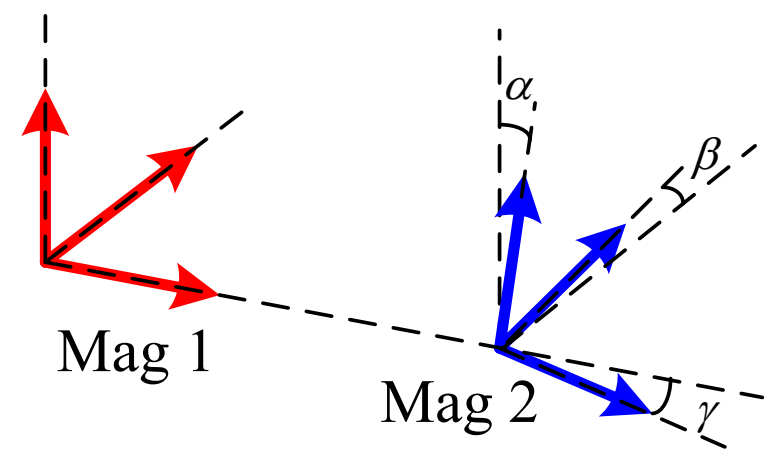

Figure 1. Misalignment error between magnetometers.

\section{Experiment}

\subsection{Experimental system}

The experimental system mainly contains a gradiometer, proton magnetometer, nonmagnetic rotation equipment and portable computer. The gradiometer consists of two fluxgate magnetometers, which is connected by an aluminium frame. There are installation interstice positions in the aluminium frame for magnetometers. Magnetometers are fixed by aluminium bolts. In order to guarantee little misalignment error between magnetometers, the installation interstices are parallel. The distance between magnetometers is $20 \mathrm{~cm}$ (Figure 2). Nonmagnetic rotation equipment is used to rotate the gradiometer. Soft material is a steel tube, and hard material is an annular magnet. The collection and process software is STL GradMag and Matlab respectively. Proton magnetometer is used to measure the true value of the magnetic field scalar. A site with stable magnetic field is chose as the calibration position. The gradiometer fixed on nonmagnetic rotation equipment is rotated symmetrically to get a batch of measurements.

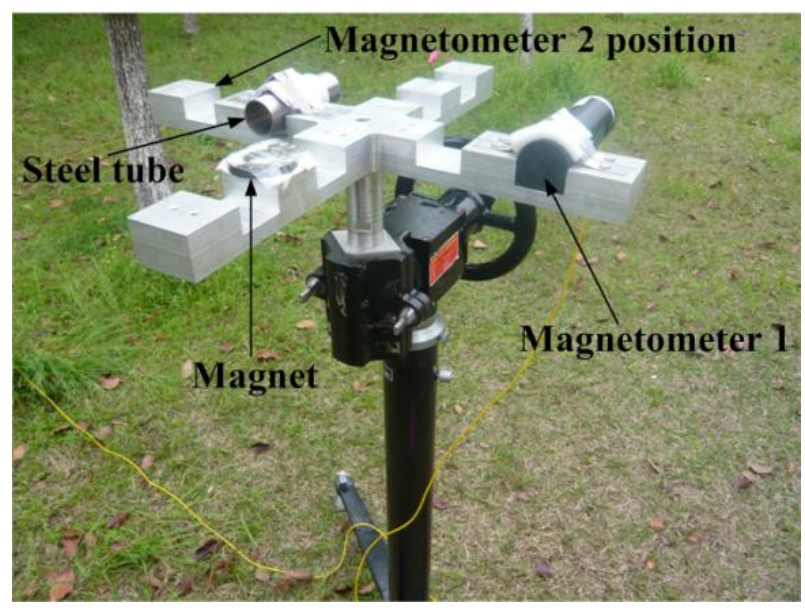

Figure 2. Gradiometers and experimental system.

\subsection{Compensation results of magnetometers}

Two magnetometers obtain a batch of measurements after the rotation of gradiometer. Firstly, magnetometers should be compensated using the scalar compensation 
method. The true intensity of geomagnetic field measured by proton magnetometer is about $48193 \mathrm{nT}$, and the magnetometers output intensity should be the same value no matter how to change the attitude. Thus, the integrated compensation performance can be evaluated.

The two magnetometers are compensated by solving nonlinear equations after the rotation. After compensation, RMS error of the first magnetometer is reduced from $1148.9 \mathrm{nT}$ to $2.6 \mathrm{nT}$ (Figure 3). The maximum error is reduced from $2141.1 \mathrm{nT}$ to $5.1 \mathrm{nT}$. The calibration result of the second magnetometer is similar (Figure 4). After calibration, RMS error of the second magnetometer is reduced from $1355.5 \mathrm{nT}$ to $2.6 \mathrm{nT}$. The maximum error is reduced from $2363.1 \mathrm{nT}$ to $6.9 \mathrm{nT}$.

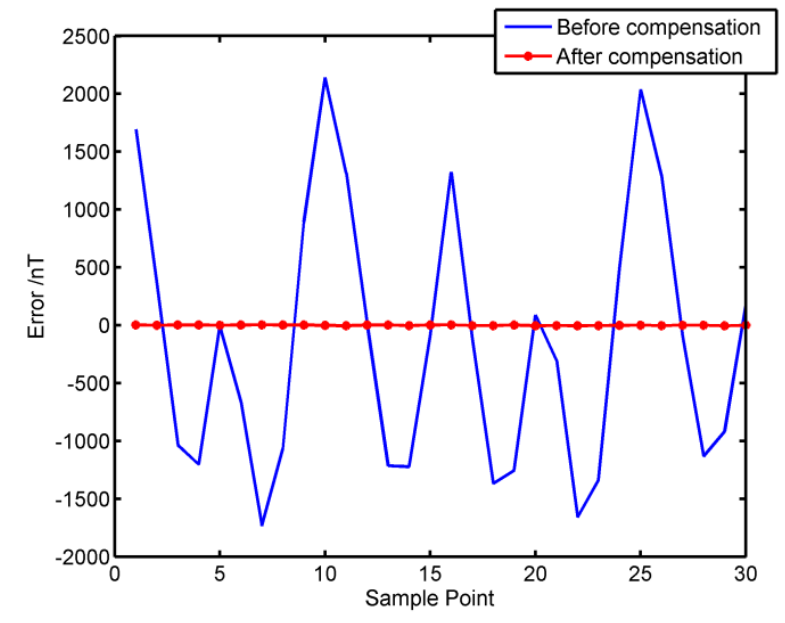

Figure 3. Compensation result of magnetometer 1 .

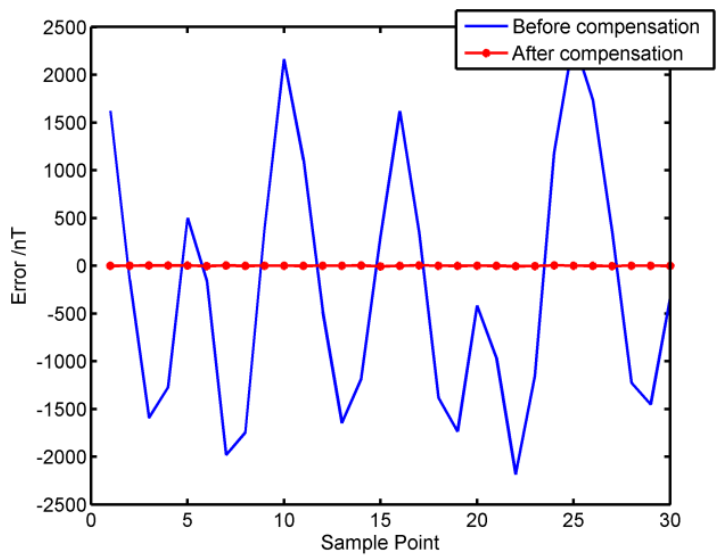

Figure 4. Compensation result of magnetometer 2.

\subsection{Calibration results of misalignment}

After the compensation of magnetometers, misalignment should be calibrated. There are two situations to analyze the calibration results of misalignment. (1) Misalignment calibration without distortion field compensation. (2) Misalignment calibration after distortion field compensation.

Figure 5 shows misalignment calibration result without distortion field compensation. Figure 5 (a), (c) and (e) show misalignment error before calibration, which reach 1300 nT. Figure 5 (b), (d) and (f) show misalignment error after calibration, which are reduced to about $750 \mathrm{nT}$. The details are shown in Table 1. It shows that the calibration accuracy is influenced without distortion field compensation, which should be improved. Figure 6 shows misalignment calibration result after distortion field compensation. Figure 6 (b), (d) and (f) show misalignment error after calibration, which are reduced to about $22 \mathrm{nT}$. Also, the details are shown in Table I . After calibration, it is obvious that the accuracy is improved by one order. The estimated misalignment angles are $\alpha=-0.7364^{\circ}, \beta=-0.0046^{\circ}, \gamma=0.0888^{\circ}$.

Table 1. Calibration result of the gradiometer in two situations.

\begin{tabular}{|l|c|c|c|}
\hline & $\mathrm{X}$ axis (nT) & Y axis (nT) & $\mathrm{Z}$ axis (nT) \\
\hline $\begin{array}{l}\text { Before } \\
\text { Calibration }\end{array}$ & 888.4 & 1292.6 & 168.9 \\
\hline Situation 1 & 421.4 & 741.3 & 124.7 \\
\hline Situation 2 & 15.3 & 22.1 & 9.9 \\
\hline
\end{tabular}

(a)

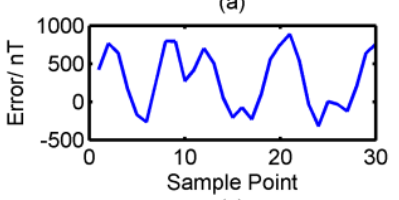

(c)

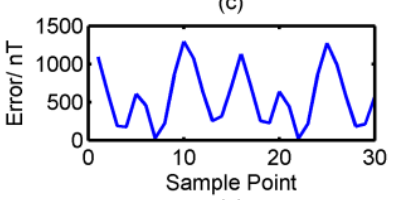

(e)

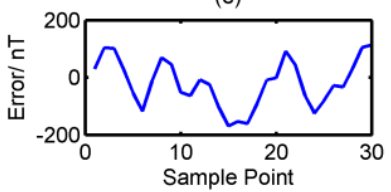

(b)
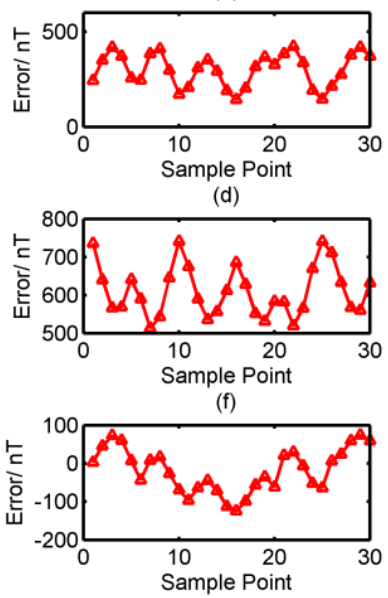

Figure 5. Calibration result of misalignment error without distortion field compensation.
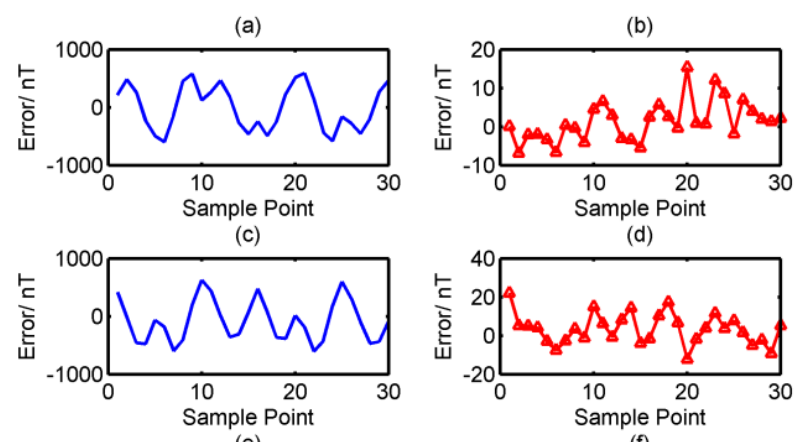

(d)

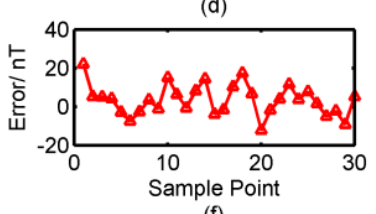

(f)
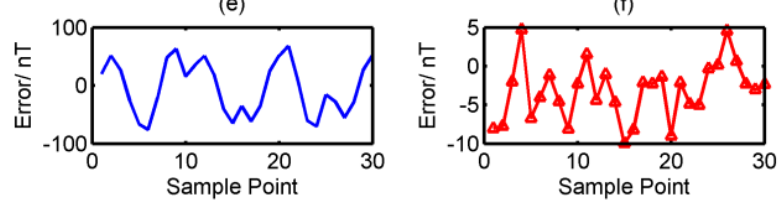

Figure 6. Calibration result of misalignment error after distortion field compensation. 


\subsection{Calibration and compensation in other situations}

In order to show the effectiveness of the proposed method, another situation is calibrated and compensated with different distortion field. In this situation, bigger soft material and hard material are deployed around the gradiometer (soft-iron 2 and hard-iron 2), which is shown in Figure 7. Similarly, the gradiometer is rotated symmetrically in 3D. Compensation performance of the first magnetometer is shown in Fig 8. After compensation, distortion field RMS error is reduced from $1891.4 \mathrm{nT}$ to $4.1 \mathrm{nT}$. The RMS error of the second magnetometer is reduced from $1516.4 \mathrm{nT}$ to $5.4 \mathrm{nT}$. As shown in Fig 9, after calibration, misalignment error is reduced to about $25 \mathrm{nT}$, thus proving the effectiveness of the proposed method.

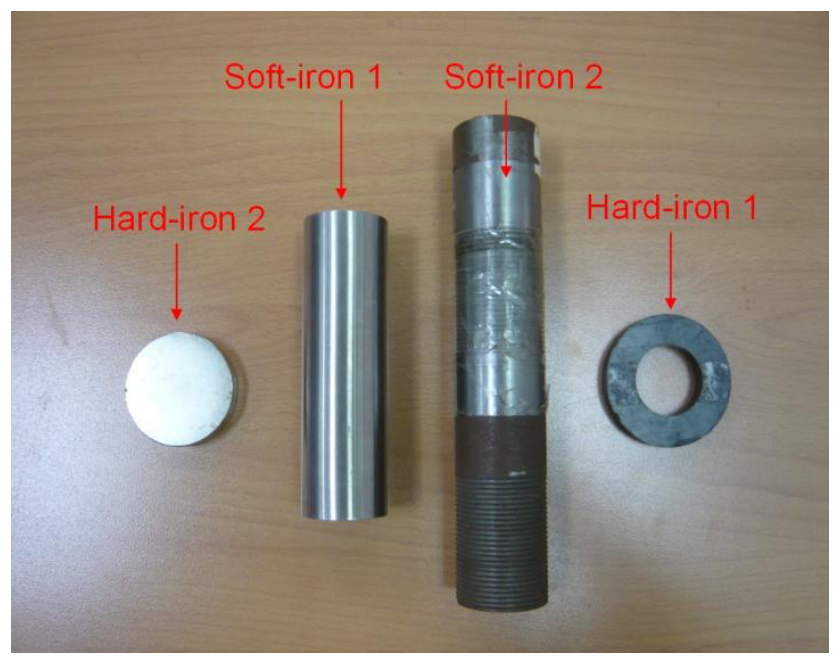

Figure 7. Different soft-material and hard-material.

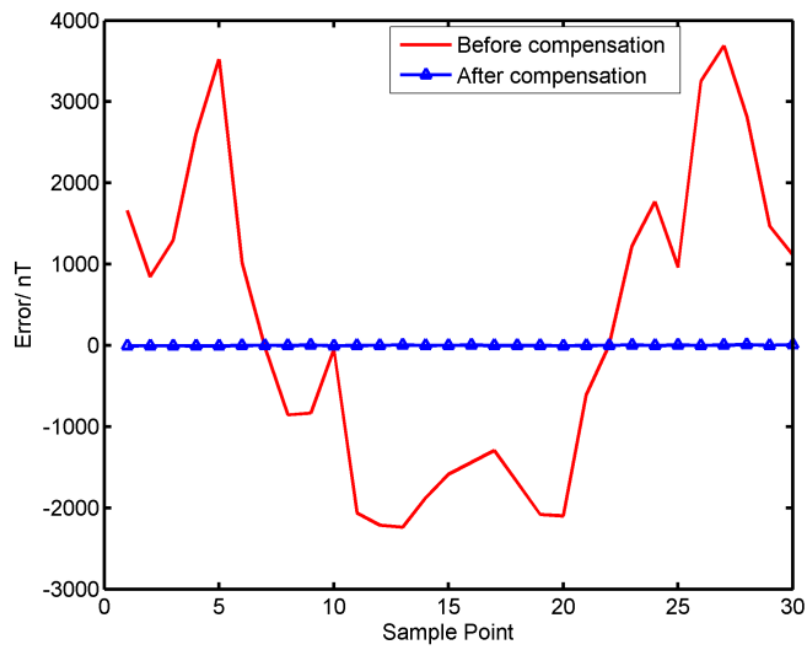

Figure 8. Compensation result of magnetometer 1 when bigger distortion fields are deployed.
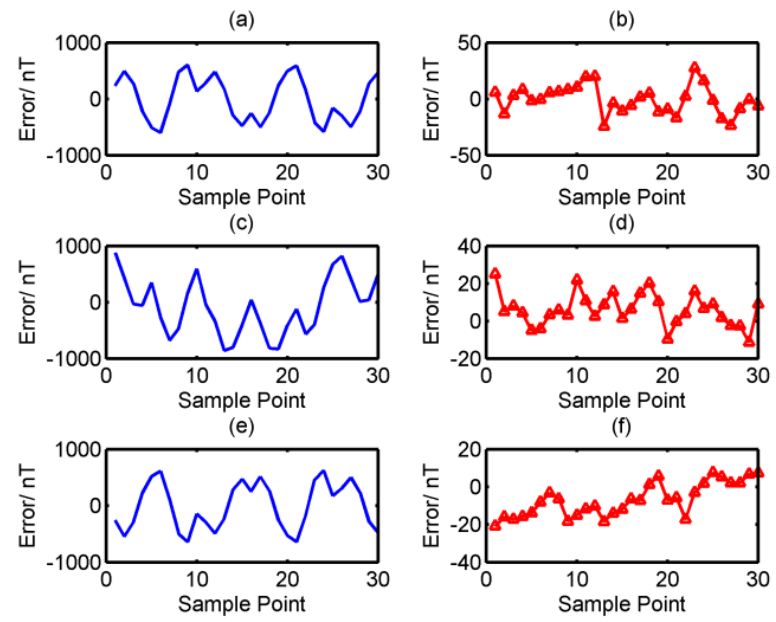

Figure 9. Calibration result of misalignment error when bigger distortion fields are deployed.

\subsection{The improvement in magnetic object remote sensing}

A magnetic abnormity object is measured by the gradiometer. There are 15 measurement positions, and the maximal distance is about 7 meters. Figure 10 shows the measured magnetic abnormity grads. Before calibration and compensation, system error reaches five hundreds of nT. After integrated compensation, there is little magnetic signal when object is removed far away. Magnetic abnormity grads become stronger when the object moves closer to the gradiometer. Thus, the magnetic abnormity grads are measured accurately after calibration and compensation.

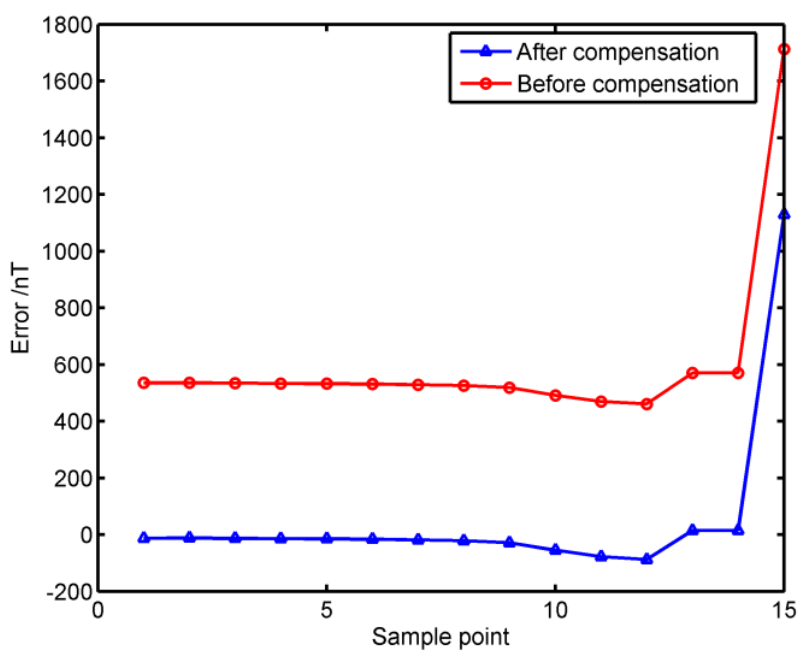

Figure 10. Magnetic abnormity grads measurement results.

\section{Conclusions}

Different scale factors, bias errors, nonorthogonality, misalignment and distortion field influence the accuracy of gradiometer. The error are calibrated and compensated by solving nonlinear equations. The accuracy is improved 
by at least one order. The calibration and compensation are still good when bigger distortion field are deployed. In addition, magnetic abnormity grads measurement results are compared. Thus, experimental results show that the proposed method can improve the accuracy of gradiometer, which is useful for magnetic object remote sensing.

\section{Acknowledgment}

This research is sponsored by National Natural Science Fund of China (Grant No. 51175507) and Young Scientist Fund of NINT (Grant No. KJYF3S01).

\section{References}

1. Y. Huang, L. H. Wu, F. Sun, IEEE Geosci. Remote. S. 11 (2013).

2. J. M. G. Merayo, P. Brauer, F. Primdahl, Sensors and Actuators A 120 (2005).

3. H. F. Pang, M. C. Pan, C. B. Wan, J. F. Chen, X. J. Zhu, F.L. Luo, IEEE Trans. Geosci. Remote Sens. 52 (2014).

4. H. F. Pang, D. X. Chen, M. C. Pan, Q. Zhang, S. T. Luo, J. Li, F. L. Luo, Sensor letters, 18 (2013).

5. D. Gebre-Egziabher, G. H. Elkaim, J. D. Powell, B. W. Parkinson, J. Aerospace. Eng. 19 (2006).

6. C. C. Foster, G. H. Elkaim, IEEE. T. Aero. Elec. Sys. 44 (2008).

7. D. G. Egziabh, J. Aircraft. 44 (2007).

8. J. L. Crassidis, K. L. Lai, R. R. Harman, J. Guidance Control Dynam. 28 (2005).

9. J. M. G. Merayo, P. Baruer, F. Primdahl, et al., Meas. Sci. Technol. 11 (2000).

10. J. M. G. Merayo, F. Primdahl, P. Brauer, et al., Sensors and Actuators A 89 (2001).

11. T. Pylvaninen, Appl. Math. Model. 32 (2008).

12. H. F. Pang, S. T. Luo, Q. Zhang, J. Li, D. X. Chen, M. C. Pan, F. L. Luo, Meas. Sci. Technol. 24 (2013).

13. H. F. Pang, J. Li, D. X Chen, M. C. Pan, S. T. Luo, Q. Zhang, F. L. Luo, Measurement 46 (2013).

14. J. Včelák, J. Appl. Phys. 99 (2006). 